\title{
Extended Poisson-Log-Logistic Distribution
}

Jehan. A. Almamy

Correspondence: Management Information System Department, Taibah University, Saudi Arabia.

E-mail: jmamy@ taibahu.edu.sa

Received: November 20, 2018 Accepted: December 27, 2018 Online Published: January 15, 2019

doi:10.5539/ijsp.v8n2p56

URL: https://doi.org/10.5539/ijsp.v8n2p56

\begin{abstract}
In this work, we introduce a new Poisson-log-logistic distribution with a physical interpretation and some applications. Some essential properties are derived. Modeling of four real data sets are provided to illustrate the wide applicability of the new model in differnt fields like finance, reliability, economy and medicine. The new compound model is better than other well-known competitive models which have at least the same number of parameters.
\end{abstract}

Keywords: log-logistic distribution, generating function, moments, truncated poisson, maximum likelihood

\section{Introduction and Physical Motivation}

The cumulative distribution function (CDF) of Burr type XII (BXII) is given as

$$
G_{(\alpha, \beta)}(x)=1-\frac{1}{\left(x^{\alpha}+1\right)^{\beta}},
$$

both $\alpha$ and $\beta$ are shape parameters (for more details about the model of BXII and other related models see Tadikamalla (1980), Rodriguez (1977), Burr and Cislak (1968), Burr (1942 and 1973)).

Setting $\beta=1$ we obtain the well known one parameter log-logistic (LL) model

$$
G_{(\alpha)}(x)=1-\frac{1}{x^{\alpha}+1} .
$$

Scale parameter can easily be added for getting other versions of the LL models as follows

$$
G_{(\alpha, \phi)}(x)=1-\frac{1}{\left(\frac{x}{\phi}\right)^{\alpha}+1} \text { and } G_{(\alpha, \phi)}(x)=1-\frac{1}{\frac{x^{\alpha}}{\phi}+1},
$$

the corresponding probability density function (PDF) of $G_{(\alpha)}(x)$ is given by

$$
g_{(\alpha)}(x)=\frac{\alpha x^{\alpha-1}}{\left(x^{\alpha}+1\right)^{2}} .
$$

Upon following Yousof et al. (2016), we propose a new model called the Burr type X LL (BXLL) model defined by the CDF given by

$$
H_{(\vartheta, \alpha)}(x)=\left(1-\mathrm{e}^{-x^{2 \alpha}}\right)^{\vartheta}
$$

where $\vartheta>0$ is a shape parameter. Suppose that we have a system has $N$ subsystems functioning independently at a given time where $N$ has zero truncated Poisson (ZTP) distribution with parameter $\lambda$. The probability mass function (PMF) of $N$ is given by

$$
\left.p_{\mathrm{ZTP}}^{(\lambda)}(N=n)\right|_{(n=1,2, \ldots)}=\frac{\mathrm{e}^{-\lambda} \lambda^{n}}{n !\left(1-\mathrm{e}^{-\lambda}\right)} .
$$

Note that for ZTP random variable (r.v.), the expected value $\mathbf{E}(N \mid \lambda)$ and the variance $\operatorname{V} \alpha r(N \mid \lambda)$ are, respectively, given by $\mathbf{E}(N \mid \lambda)=\lambda /\left(1-\mathrm{e}^{-\lambda}\right)$ and $\operatorname{Vor}(N \mid \lambda)=\frac{(1+\lambda) \lambda}{1-\mathrm{e}^{-\lambda}}-\left[\frac{\lambda}{1-\mathrm{e}^{-\lambda}}\right]^{2}$.

Assume that the failure time of each subsystem has the $\operatorname{BXLL}(\vartheta, \alpha)$. Let $Y_{i}$ denote the failure time of the $i^{\text {th }}$ subsystem, let

$$
X=\min \left\{Y_{1}, Y_{2}, \cdots Y_{N-1}, Y_{N}\right\}
$$

Then, the conditional CDF of $X \mid N$ is

$$
F(x \mid N)=1-\operatorname{Pr}(X>x \mid N)=1-\left[1-H_{(\vartheta, \alpha)}(x)\right]^{N} .
$$




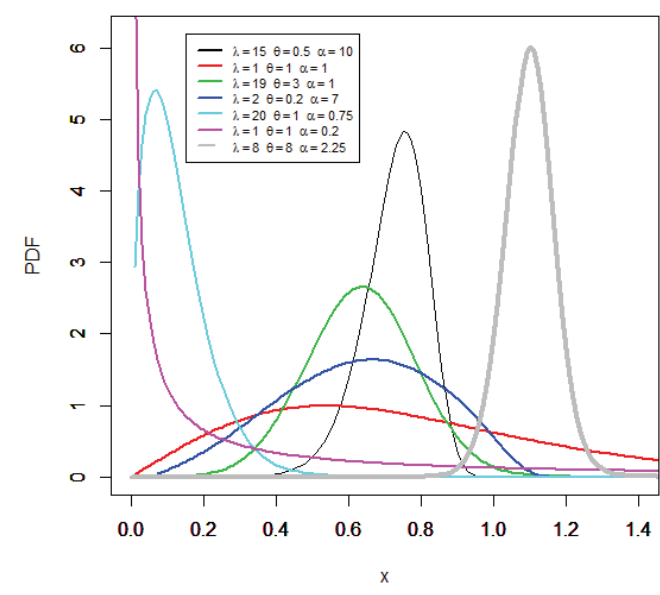

(a)

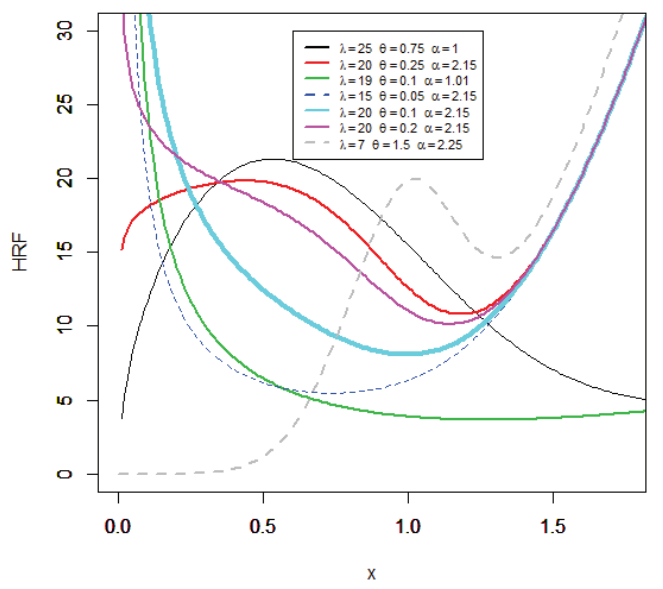

(b)

Figure 1. PDFs and HRFs plots for the PBXLL model

So, the unconditional CDF of the Poisson Burr type X log-logistic (PBXLL) can be expressed as

$$
F_{(\lambda, \vartheta, \alpha)}(x)=\frac{1-\mathrm{e}^{-\lambda\left(1-\mathrm{e}^{-x^{2 \alpha}}\right)^{\vartheta}}}{1-\mathrm{e}^{-\lambda}}
$$

with the corresponding PDF as

$$
f_{(\lambda, \vartheta, \alpha)}(x)=2 \alpha \lambda \vartheta x^{2 \alpha-1}\left(1-\mathrm{e}^{-\lambda}\right)^{-1}\left(1-\mathrm{e}^{-x^{2 \alpha}}\right)^{\vartheta-1} \mathrm{e}^{-x^{2 \alpha}-\lambda\left(1-\mathrm{e}^{-x^{2 \alpha}}\right)^{\vartheta}} .
$$

The PBXLL reduces to Poisson Rayleigh log-logistic when $\vartheta=1$. After some algebra the PDF of the PBXLL can be expressed as

$$
f_{(\lambda, \vartheta, \alpha)}(x)=\sum_{r=0}^{\infty} v_{r} \pi_{[\alpha,(1+r)]}(x)
$$

where

$$
v_{r}=\sum_{h, w, m, i=0}^{\infty} \frac{2 \vartheta \lambda^{1+m}(-1)^{h+r+m+i}(i+1)^{h} \Gamma(3+2 h+w) \Gamma((1+m) \vartheta) \Gamma(2(1+h)+w)}{h ! w ! i !(1+r) !\left(1-\mathrm{e}^{-\lambda}\right) \Gamma(3+2 h) \Gamma(2 h+w+2-r) \Gamma((1+m) \vartheta-i)},
$$

and $\pi_{[\alpha,(1+r)]}(x)$ is the LL density with parameters $\alpha$ and $(1+r)$. Similarly, the CDF of the PBXLL can also be expressed as

$$
F_{(\lambda, \vartheta, \alpha)}(x)=\sum_{r=0}^{\infty} v_{r} \Pi_{[\alpha,(1+r)]}(x)
$$

where $\Pi_{[\alpha,(1+r)]}(x)$ is the LL CDF with parameters $\alpha$ and $(1+r)$. The hazard rate function (HRF) of the new model can be calculated from $f_{(\lambda, \vartheta, \alpha)}(x) /\left[1-F_{(\lambda, \vartheta, \alpha)}(x)\right]$. The PBXLL density can be left-skewed, right-skewed, unimodal and symmetric (see Figure 1(a)) while the PBXLL HRF can be bathtub or unimodal or unimodal then bathtub or decreasing or unimodal then increasing (see Figure 1(b)).

The quantile function $(\mathrm{QF})$ of $X$, where $X \sim \operatorname{PBXLL}(\lambda, \vartheta, \alpha)$, is obtained by inverting (5) as

$$
Q(u)=\left\{\left[1-\left(1+\left\{-\ln \left[1-\left(\frac{-\ln \left\{1-u\left(1-\mathrm{e}^{-\lambda}\right)\right\}}{\lambda}\right)^{\frac{1}{\vartheta}}\right]\right\}^{\frac{1}{2}}\right)\right]-1\right\}^{\frac{1}{\alpha}}
$$

Simulating the PBXLL r.v. is straightforward. If $U$ is a uniform variate on the unit interval $(0,1)$, then the r.v. $X=Q(U)$ follows (5). 
The $n$th ordinary moment of $X$, say $\mu_{r}^{\prime}$, follows from (7) as

$$
\left.\mu_{n}^{\prime}\right|_{[n<(1+r) \alpha \beta]}=\mathbf{E}\left(X^{n}\right)=\sum_{r=0}^{\infty} v_{r}(1+r) B\left(1+\frac{n}{\alpha},(1+r)-\frac{n}{\alpha}\right),
$$

Setting $n=1$ in (9) gives the mean of $X$. The $n$th incomplete moment of $X$ is defined by $m_{n}(t)=\int_{-\infty}^{t} x^{r} f(x) d x$. We can write from (7)

$$
\left.m_{n}(t)\right|_{[n<(1+r) \alpha \beta]}=\sum_{r=0}^{\infty} v_{r}(1+r) B\left(t^{\alpha} ; 1+\frac{n}{\alpha},(1+r)-\frac{n}{\alpha}\right),
$$

where

$$
\int_{0}^{\infty}(1+x)^{-(\alpha+\beta)} x^{\alpha-1} d x=B(\alpha, \beta)
$$

and

$$
\int_{0}^{t}(1+x)^{-(\alpha+\beta)} x^{\alpha-1} d x=B(t ; \alpha, \beta)
$$

are beta and incomplete beta functions from the second type, respectively.

\section{Parameter Estimation}

The log-likelihood function $\left(\ell_{n}(\phi)\right)$ for $\phi$ is given by

$$
\begin{aligned}
\ell_{n}(\phi)= & n \log (2)+n \log (\vartheta)+n \log (\lambda)+n \log (\alpha)-n \log \left(-\mathrm{e}^{-\lambda}+1\right) \\
& +(-1+\alpha) \sum_{i=1}^{n} \log x_{i}+\sum_{i=1}^{n} \log s_{i}+\sum_{i=1}^{n} \log \left(1-\frac{1}{s_{i}}\right) \\
& -\lambda \sum_{i=1}^{n}\left(1-z_{i}\right)^{\vartheta}+\sum_{i=1}^{n} \log z_{i}+(\vartheta-1) \sum_{i=1}^{n} \log \left(1-z_{i}\right)
\end{aligned}
$$

where

$$
s_{i}=x^{\alpha}+1 \text { and } z_{i}=\exp \left[-\left(s_{i}-1\right)^{2}\right] .
$$

The $\ell_{n}(\boldsymbol{\Phi})$ in (10) can be numerically maximized via SASor R or 0x programs. The components of the score vector, $\mathbf{U}(\phi)=\frac{\partial \ell}{\partial \phi}=\left(\frac{\partial \ell_{n}(\boldsymbol{\Phi})}{\partial \lambda}, \frac{\partial \ell_{n}(\boldsymbol{\Phi})}{\partial \vartheta}, \frac{\partial \ell_{n}(\boldsymbol{\Phi})}{\partial \alpha}\right)^{\top}$ are easily to be derived.

\section{Applications}

For the four data sets, we will compare the PBXLL distribution with other well-known generalizations of the LL model such as the BXII, Zografos-Balakrishnan BXII (ZBBXII), Marshall-Olkin BXII (MOBXII), the Five Parameters beta BXII (FBBXII), BBXII, Beta exponentiated BXII (BEBXII), Five Parameters Kumaraswamy BXII (FKumBXII), Topp Leone BXII (TLBXII) and KumBXII distributions (for more details about the competitive models see Altun et al. 2018 a, b and Yousof et al. 2018 a, b). Data Set I called breaking stress data. This data set consists of 100 observations of breaking stress of carbon fibres (in Gba) given by Nichols and Padgett (2006). Data Set II called survival times. In this application, we work with the survival times (in days) of 72 guinea pigs infected with virulent tubercle bacilli, originally observed and reported by Bjerkedal (1960). Data Set III called taxes revenue data. The actual taxes revenue data (in 1000 million Egyptian pounds). Data set IV called leukaemia data. This real data set gives the survival times, in weeks, of 33 patients suffering from acute Myelogeneous Leukaemia (see the appendix).

The total time test (T.T.T.) plots for the four real data sets is presented in Figure 2. This plot indicates that the empirical HRFs of the four data sets are increasing, increasing, increasing and $\mathbf{U}$-shaped (for more details about the T.T.T. see Aarset (1987)).

We will consider the following goodness-of-fit statistics:

1-Akaike Information Criterion $\left(\mathrm{AI}_{\mathrm{c}}\right)$;

2-Bayesian Information Criterion $\left(\mathrm{BI}_{\mathrm{c}}\right)$;

3-Hannan-Quinn Information Criterion $\left(\mathrm{HQI}_{\mathrm{c}}\right.$ ); 


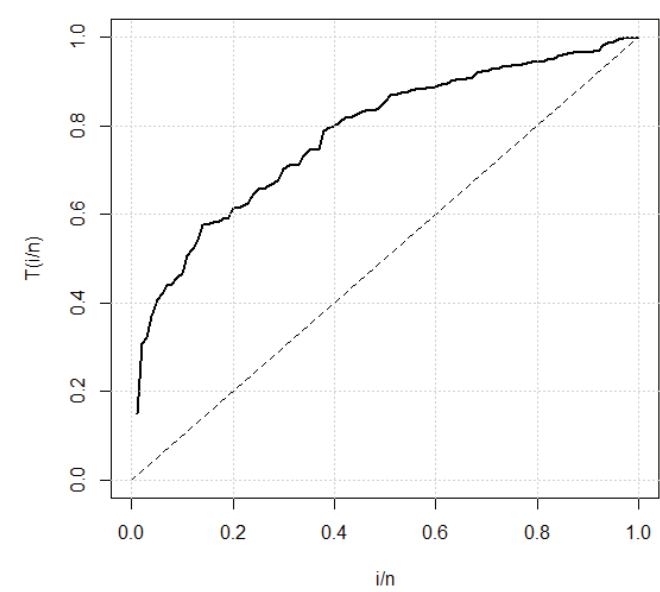

Data set $\mathbf{I}$.

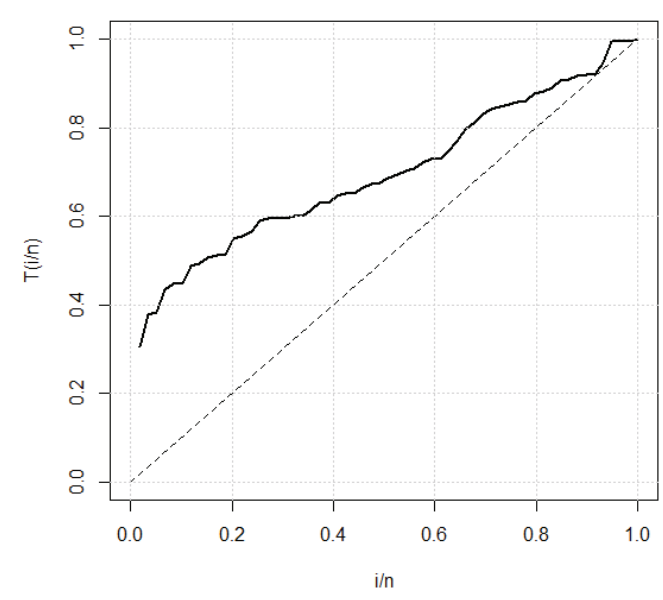

Data set III.

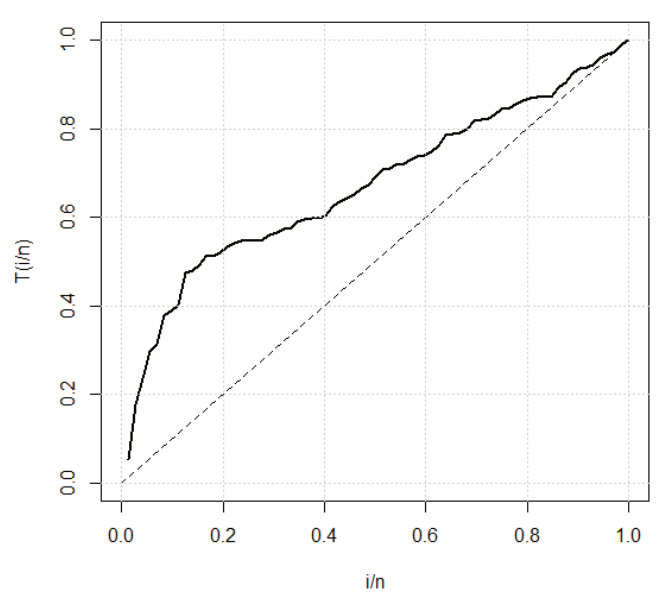

Data set II.

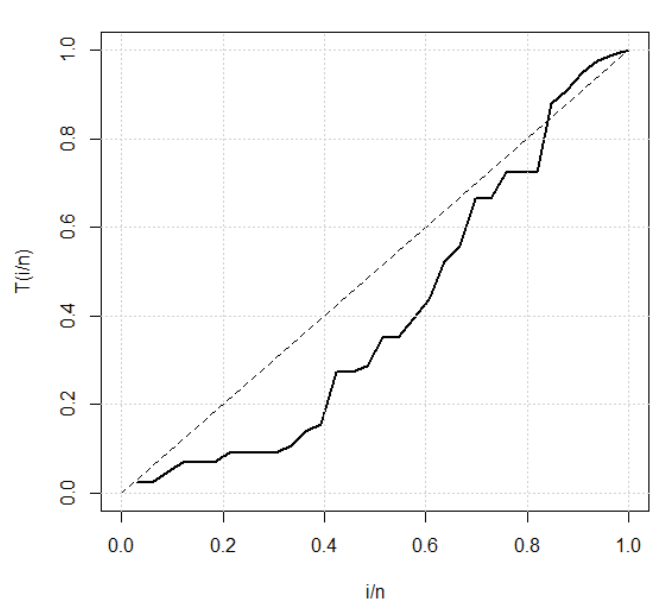

Data set IV.

Figure 2. T.T.T. plots

4-Consistent Akaike Information Criterion $\left(\mathrm{CAI}_{\mathrm{c}}\right)$, where

$$
\begin{aligned}
\mathrm{AI}_{\mathrm{c}} & =2[-\ell(\widehat{\phi})+K], \mathrm{BI}_{\mathrm{c}}=2\left[-\ell(\widehat{\phi})+\frac{1}{2} K \log (n)\right], \\
\mathrm{HQI}_{\mathrm{c}} & =2\{-\ell(\widehat{\phi})+K \log [\log (n)]\} \text { and } \mathrm{CAI}_{\mathrm{c}}=2\left[-\ell(\widehat{\phi})+\frac{K n}{n-K-1}\right],
\end{aligned}
$$

where $K$ is the number of parameters, $n$ is the sample size, $-2 \ell(\widehat{\phi})$ is the maximized log-likelihood. Generally, the smaller these statistics are, the better the fit. Table 1, 2, 3 and 4 gives the MLEs and standard errors, confidence interval (in parentheses) with $\mathrm{AI}_{c}, \mathrm{BI}_{c}, \mathrm{CAI}_{\mathrm{c}}$ and $\mathrm{HQI}_{\mathrm{c}}$ values for the data set $\mathbf{I}$, II, III and $\mathbf{I V}$ respectively. .Figures 3, 4 and 5 gives the estimated density, the estimated CDF and the estimated HRF for the four data sets. Figure 6 gives the p-p plot for the four data sets. Figure 7 gives the Kaplan-Meier survival plots for the four data sets. From these two figure we note the new model could be chosen to fit these four data sets. Based on the values in Tables 1,2, 3 and 4 and Figure 3-7 the PBXLL model provides the best fits as compared to other BXII models in the four applications with small values for $\mathrm{BI}_{\mathrm{c}}$, $\mathrm{AI}_{\mathrm{c}}, \mathrm{CAI}_{\mathrm{c}}$ and $\mathrm{HQI} \mathrm{I}_{\mathrm{c}}$. 
Table 1. MLEs and standard errors, confidence interval (in parentheses) with $\mathrm{AI}_{\mathrm{c}}, \mathrm{BI}_{\mathrm{c}}, \mathrm{CAI}_{\mathrm{c}}$ and $\mathrm{HQI} \mathrm{I}_{\mathrm{c}}$ values for the data set I

\begin{tabular}{|c|c|c|}
\hline Model & $\widehat{\lambda}, \widehat{\vartheta}, \widehat{\alpha}, \widehat{\beta}, \widehat{\gamma}$ & $\mathrm{AI}_{\mathrm{c}}, \mathrm{BI}_{\mathrm{c}}, \mathrm{CAI}_{\mathrm{c}}, \mathrm{HQI}_{\mathrm{c}}$ \\
\hline BXII & $\begin{array}{c}\text { 一,一, 5.941, } 0.187,- \\
\text {-, },(1.279),(0.044),- \\
-,-,(3.43,8.45),(0.10,0.27),-\end{array}$ & $382.94,388.15,383.06,385.05$ \\
\hline MOBXII & $\begin{array}{c}\text {-, - } 1.192,4.834,838.73 \\
-,-,(0.952),(4.896),(229.34) \\
-,-, 0,3.06),(0,14.43),(389.22,1288.24)\end{array}$ & $305.78,313.61,306.03,308.96$ \\
\hline TLBXII & $\begin{array}{c}\text {-, }, 1.350,1.061,13.728 \\
\text {-, }, 0.378),(0.384),(8.400) \\
-,-(0.61,2.09),(0.31,1.81),(0,30.19)\end{array}$ & $323.52,331.35,323.77,326.70$ \\
\hline KumBXII & $\begin{array}{c}48.103,79.516,0.351,2.730,- \\
(19.348),(58.186),(0.098),(1.077),- \\
(10.18,86.03),(0,193.56),(0.16,0.54),(0.62,4.84),-\end{array}$ & $303.76,314.20,304.18,308.00$ \\
\hline BBXII & $\begin{array}{c}359.683,260.097,0.175,1.123,- \\
(57.941),(132.213),(0.013),(0.243),- \\
(246.1,473.2),(0.96,519.2),(0.14,0.20),(0.65,1.6),-\end{array}$ & $305.64,316.06,306.06,309.85$ \\
\hline BEBXII & $\begin{array}{c}0.381,11.949,0.937,33.402,1.705 \\
(0.078),(4.635),(0.267),(6.287),(0.478) \\
(0.23,0.53),(2.86,21),(0.41,1.5),(21,45),(0.8,2.6)\end{array}$ & $305.82,318.84,306.46,311.09$ \\
\hline FBBXII & $\begin{array}{c}0.421,0.834,6.111,1.674,3.450 \\
(0.011),(0.943),(2.314),(0.226),(1.957) \\
(0.4,0.44),(0.2 .7),(1.57,10.7),(1.23,2.1),(0,7)\end{array}$ & $304.26,317.31,304.89,309.56$ \\
\hline FKumBXII & $\begin{array}{c}0.542,4.223,5.313,0.411,4.152 \\
(0.137),(1.882),(2.318),(0.497),(1.995) \\
(0.3,0.8),(0.53,7.9),(0.9,9),(0,1.7),(0.2,8)\end{array}$ & $305.50,318.55,306.14,310.80$ \\
\hline ZBBXII & $\begin{array}{c}123.101,-, 0.368,139.247,- \\
(243.011),-,(0.343),(318.546),- \\
(0,599.40),-,(0,1.04),(0,763.59),-\end{array}$ & $302.96,310.78,303.21,306.13$ \\
\hline PBXLL & $\begin{array}{c}-3.21,3.67,0.56,-,- \\
1.72,1.837,0.023,-,- \\
(-6.4,0.4),(0.13,7.33),(0.52,0.6),-,-\end{array}$ & $290.6,298.4,290.8,293.7$ \\
\hline
\end{tabular}


Table 2. MLEs and standard errors, confidence interval (in parentheses) with $\mathrm{AI}_{\mathrm{c}}, \mathrm{BI}_{\mathrm{c}}, \mathrm{CAI}_{\mathrm{c}}$ and $\mathrm{HQI}_{\mathrm{c}}$ values for the data set II

\begin{tabular}{|c|c|c|}
\hline Model & $\widehat{\lambda}, \widehat{\vartheta}, \widehat{\alpha}, \widehat{\beta}, \widehat{\gamma}$ & $\mathrm{AI}_{\mathrm{c}}, \mathrm{BI}_{\mathrm{c}}, \mathrm{CAI}_{\mathrm{c}}, \mathrm{HQI}_{\mathrm{c}}$ \\
\hline BXII & $\begin{array}{c}\text {-,-, 3.102, } 0.465,- \\
\text {-, },(0.538),(0.077),- \\
\text {-, },(2.05,4.16),(0.31,0.62),-\end{array}$ & $209.60,214.15,209.77,211.40$ \\
\hline MOBXII & $\begin{array}{c}\text { —, - } 2.259,1.533,6.760 \\
\text { —, },(0.864),(0.907),(4.587) \\
\text {-, }(0.57,3.95),(0,3.31),(0,15.75)\end{array}$ & $209.74,216.56,210.09,212.44$ \\
\hline TLBXII & $\begin{array}{c}\text { —,-, } 2.393,0.458,1.796 \\
\text {-, },(0.907),(0.244),(0.915) \\
\text {-, },(0.62,4.17),(0,0.94),(0.002,3.59)\end{array}$ & $211.80,218.63,212.15,214.52$ \\
\hline KumBXII & $\begin{array}{c}14.105,7.424,0.525,2.274,- \\
(10.805),(11.850),(0.279),(0.990),- \\
(0,35.28),(0.30 .65),(0,1.07),(0.33,4.21),-\end{array}$ & $208.76,217.86,209.36,212.38$ \\
\hline BBXII & $\begin{array}{c}2.555,6.058,1.800,0.294,- \\
(1.859),(10.391),(0.955),(0.466),- \\
(0,6.28),(0,26.42),(0,3.67),(0,1.21),-\end{array}$ & $210.44,219.54,211.03,214.06$ \\
\hline BEBXII & $\begin{array}{c}1.876,2.991,1.780,1.341,0.572 \\
(0.094),(1.731),(0.702),(0.816),(0.325) \\
(1.7,2.06),(0,6.4),(0.40,3.2),(0,2.9),(0,1.21)\end{array}$ & $212.10,223.50,213.00,216.60$ \\
\hline FBBXII & $\begin{array}{c}0.621,0.549,3.838,1.381,1.665 \\
(0.541),(1.011),(2.785),(2.312),(0.436) \\
(0,1.7),(0,2.5),(0,9.3),(0,5.9),(0.8,4.5)\end{array}$ & $206.80,218.20,207.71,211.30$ \\
\hline FKumBXII & $\begin{array}{c}0.558,0.308,3.999,2.131,1.475 \\
(0.442),(0.314),(2.082),(1.833),(0.361) \\
(0,1.4),(0,0.9),(0,3.1),(0,5.7),(0.76,2.2)\end{array}$ & $206.50,217.90,207.41,211.00$ \\
\hline PBXLL & $\begin{array}{c}1.5,4.34,0.45,-,- \\
(2.2),(1.7),(0.1),-,- \\
(0,5.9),(0.94,7.7),(0.25,0.65),-,-\end{array}$ & 204.8, 211.6, 205.1, 207.5 \\
\hline
\end{tabular}


Table 3. MLEs and standard errors, confidence interval (in parentheses) with $\mathrm{AI}_{\mathrm{c}}, \mathrm{BI}_{\mathrm{c}}, \mathrm{CAI}_{\mathrm{c}}$ and $\mathrm{HQI} \mathrm{I}_{\mathrm{c}}$ values for the data set III

\begin{tabular}{|c|c|c|}
\hline Model & $\widehat{\lambda}, \widehat{\vartheta}, \widehat{\alpha}, \widehat{\beta}, \widehat{\gamma}$ & $\mathrm{AI}_{\mathrm{c}}, \mathrm{BI}_{\mathrm{c}}, \mathrm{CAI}_{\mathrm{c}}, \mathrm{HQI}_{\mathrm{c}}$ \\
\hline BXII & $\begin{array}{c}\text {-,-, 5.615, 0.072,-- } \\
\text {-,-, }(15.048),(0.194),- \\
\text {-, },(0,35.11),(0,0.45),-\end{array}$ & $518.46,522.62,518.67,520.08$ \\
\hline MOBXII & $\begin{array}{c}\text { —, - }, 8.017,0.419,70.359 \\
\text { 一, },(22.083),(0.312),(63.831) \\
\text {,-, }(0,51.29),(0,1.03),(0,195.47)\end{array}$ & $387.22,389.38,387.66,389.68$ \\
\hline TLBXII & $\begin{array}{c}\text {-, - }, 91.320,0.012,141.073 \\
\text {-, }(15.071),(0.002),(70.028) \\
\text {-,_, }(61.78,120.86)(0.008,0.02)(3.82,278.33)\end{array}$ & $385.94,392.18,386.38,388.40$ \\
\hline KumBXII & $\begin{array}{c}18.130,6.857,10.694,0.081,- \\
(3.689),(1.035),(1.166),(0.012),- \\
(10.89,25.36),(4.83,8.89),(8.41,12.98),(0.06,0.10),-\end{array}$ & $385.58,393.90,386.32,388.86$ \\
\hline BBXII & $\begin{array}{c}26.725,9.756,27.364,0.020,- \\
(9.465),(2.781),(12.351),(0.007),- \\
(8.17,45.27),(4.31,15.21),(3.16,51.57),(0.006,0.03),-\end{array}$ & $385.56,394.10,386.30,389.10$ \\
\hline BEBXII & $\begin{array}{c}2.924,2.911,3.270,12.486,0.371 \\
(0.564),(0.549),(1.251),(6.938),(0.788) \\
(1.82,4.03),(1.83,3.99),(0.82,5.72),(0,26.08),(0,1.92)\end{array}$ & $387.04,397.42,388.17,391.09$ \\
\hline FBBXII & $\begin{array}{c}30.441,0.584,1.089,5.166,7.862 \\
(91.745),(1.064),(1.021),(8.268),(15.036) \\
(0,210.26),(0,2.67),(0,3.09),(0,21.37),(0,37.33)\end{array}$ & $386.74,397.14,387.87,390.84$ \\
\hline FKumBXII & $\begin{array}{c}12.878,1.225,1.665,1.411,3.732 \\
(3.442),(0.131),(0.034),(0.088),(1.172) \\
(6.13,19.62),(0.97,1.48),(1.56,1.73),(1.24,1.58),(1.43,6.03),-\end{array}$ & $386.96,397.36,388.09,391.06$ \\
\hline PBXLL & $\begin{array}{c}-91.5,0.43,0.29,-,- \\
(0.0),(0.0),(0.0),-,- \\
-\end{array}$ & 383.9, 390.1, 384.3, 386.3 \\
\hline
\end{tabular}


Table 4. MLEs and standard errors, confidence interval (in parentheses) with $\mathrm{AI}_{\mathrm{c}}, \mathrm{BI}_{\mathrm{c}}, \mathrm{CAI}_{\mathrm{c}}$ and $\mathrm{HQI} \mathrm{I}_{\mathrm{c}}$ values for the data set IV

\begin{tabular}{|c|c|c|}
\hline Model & $\widehat{\lambda}, \widehat{\vartheta}, \widehat{\alpha}, \widehat{\beta}, \widehat{\gamma}$ & $\mathrm{AI}_{\mathrm{c}}, \mathrm{BI}_{\mathrm{c}}, \mathrm{CAI}_{\mathrm{c}}, \mathrm{HQI}_{\mathrm{c}}$ \\
\hline BXII & $\begin{array}{c}\text {-,-, 58.711,0.006,- } \\
\text {-,-, }(42.382),(0.004),- \\
\text {,-, }(0,141.78),(0,0.01),-\end{array}$ & $328.20,331.19,328.60,329.19$ \\
\hline MOBXII & $\begin{array}{c}\text { —, }, 11.838,0.078,12.251 \\
\text {-, },(4.368),(0.013),(7.770) \\
\text {-, },(0,141.78),(0,0.01),(0,27.48)\end{array}$ & $315.54,320.01,316.37,317.04$ \\
\hline TLBXII & $\begin{array}{c}\text {-, }, 0.281,1.882,50.215 \\
\text {-, },(0.288),(2.402),(176.50) \\
\text {,-, }(0,0.85),(0,6.59),(0,396.16)\end{array}$ & $316.26,320.73,317.09,317.76$ \\
\hline KumBXII & $\begin{array}{c}9.201,36.428,0.242,0.941,- \\
(10.060),(35.650),(0.167),(1.045),- \\
(0,28.912),(0,106.30),(0,0.57),(0,2.99),-\end{array}$ & $317.36,323.30,318.79,319.34$ \\
\hline BBXII & $\begin{array}{c}96.104,52.121,0.104,1.227,- \\
(41.201),(33.490),(0.023),(0.326),- \\
(15.4,176.8),(0,117.8),(0.6,0.15),(0.59,1.9),-\end{array}$ & $316.46,322.45,317.89,318.47$ \\
\hline BEBXII & $\begin{array}{c}0.087,5.007,1.561,31.270,0.318 \\
(0.077),(3.851),(0.012),(12.940),(0.034) \\
(0,0.3),(0,12.6),(1.5,1.6),(5.9,56.6),(0.3,0.4)\end{array}$ & $317.58,325.06,319.80,320.09$ \\
\hline FBBXII & $\begin{array}{c}15.194,32.048,0.233,0.581,21.855 \\
(11.58),(9.867),(0.091),(0.067),(35.548) \\
(0,37.8),(12.7,51.4),(0.05,0.4),(0.45,0.7),(0,91.5)\end{array}$ & $317.86,325.34,320.08,320.36$ \\
\hline FKumBXII & $\begin{array}{c}14.732,15.285,0.293,0.839,0.034 \\
(12.390),(18.868),(0.215),(0.854),(0.075) \\
(0,39.02),(0,52.27),(0,0.71),(0,2.51),(0,0.18)\end{array}$ & $317.76,325.21,319.98,320.26$ \\
\hline ZBBXII & $\begin{array}{c}41.973,-, 0.157,44.263,- \\
(38.787),-,(0.082),(47.648),- \\
(0,117.99),-,(0,0.32,)(0,137.65),-\end{array}$ & $313.86,318.35,314.39,315.36$ \\
\hline PBXLL & $\begin{array}{c}-0.48,6.594,0.16,-,- \\
(1.4),(2.37),(0.015),-,- \\
(-3.3,2.3),(1.8,11.4),(0.13,0.19),-,-\end{array}$ & $313.4,317.9,314.29,314.9$ \\
\hline
\end{tabular}




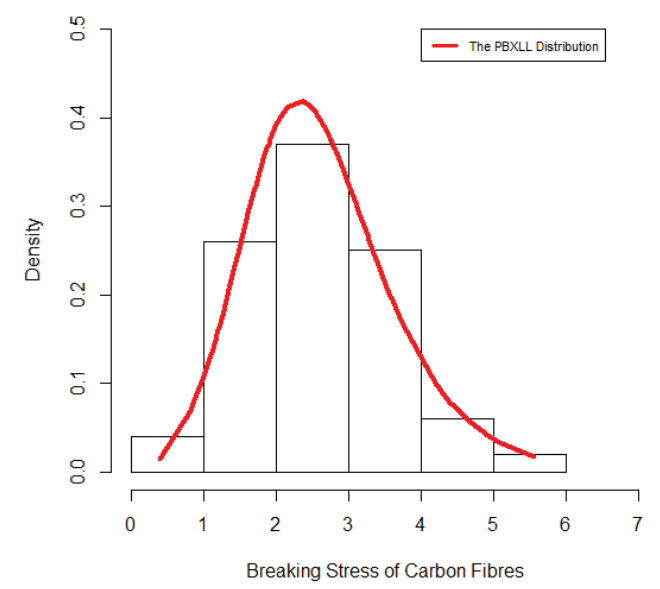

Data set $\mathbf{I}$

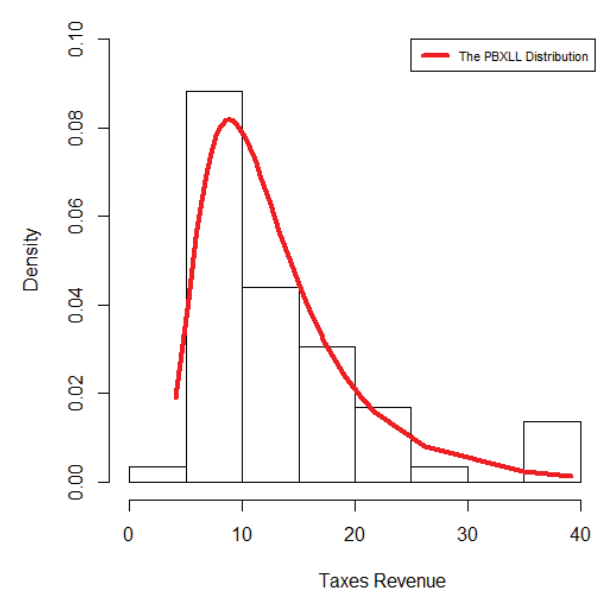

Data set III

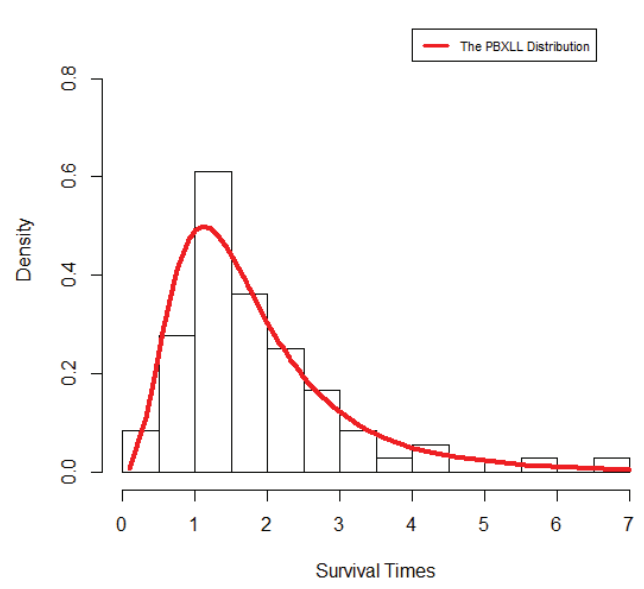

Data set II

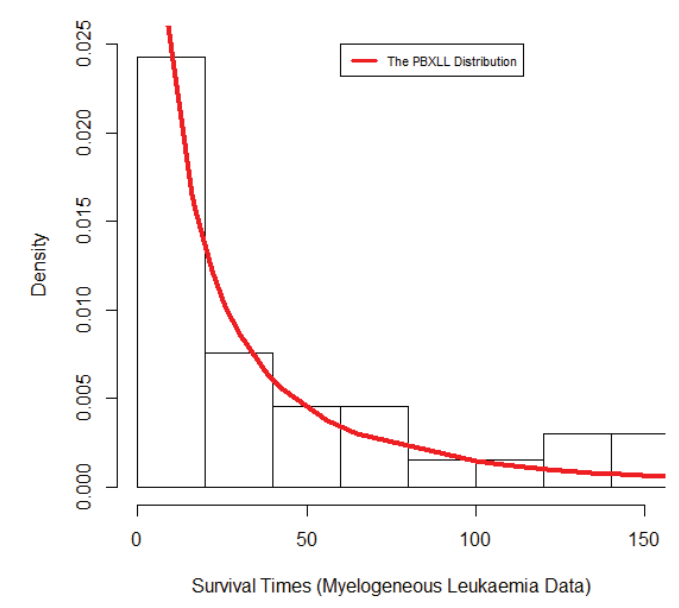

Data set IV

Figure 3. Histograms 


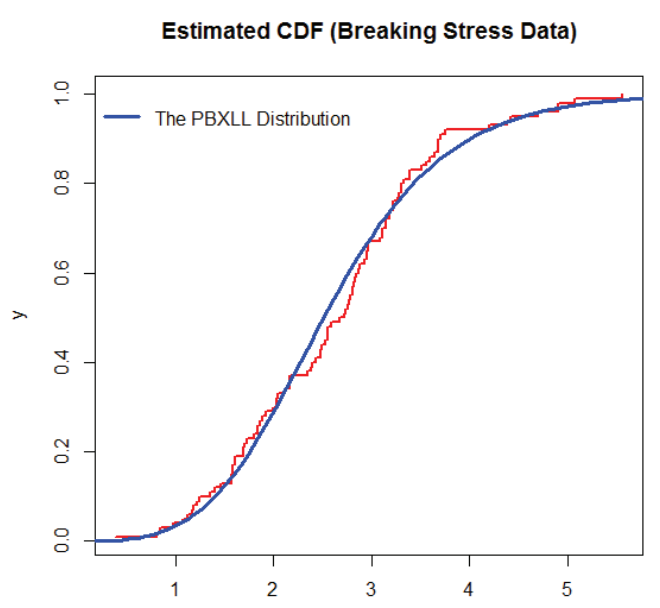

Data set $\mathbf{I}$.

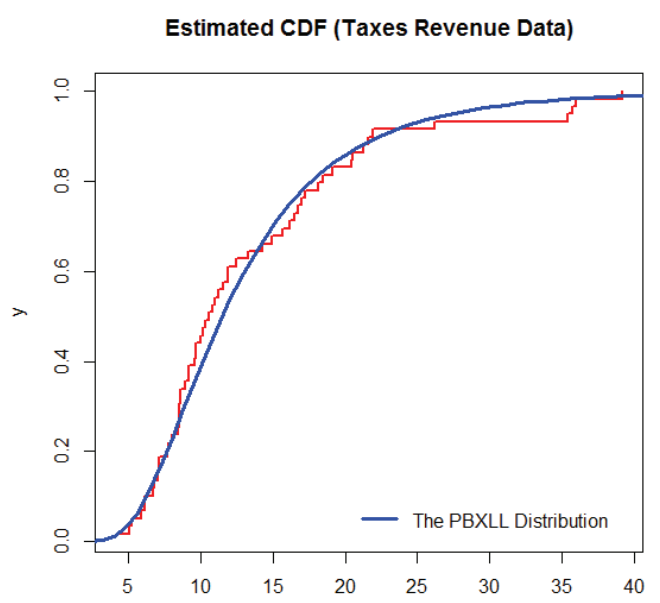

Data set III.
Estimated CDF (Survival Times Data)

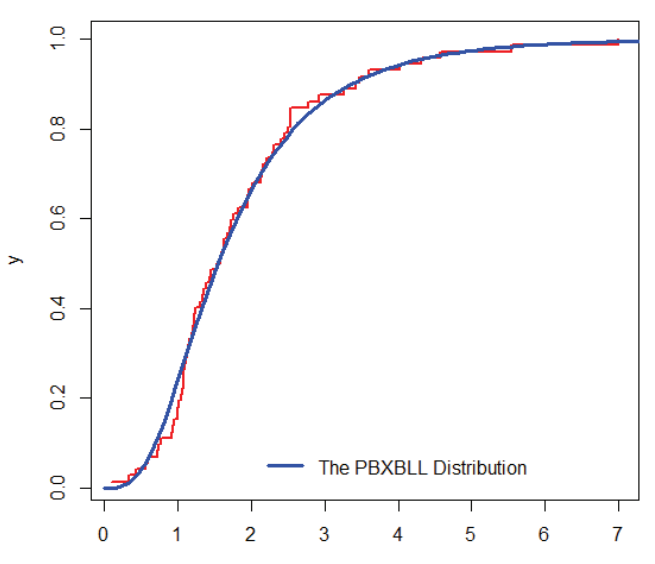

Data set II.

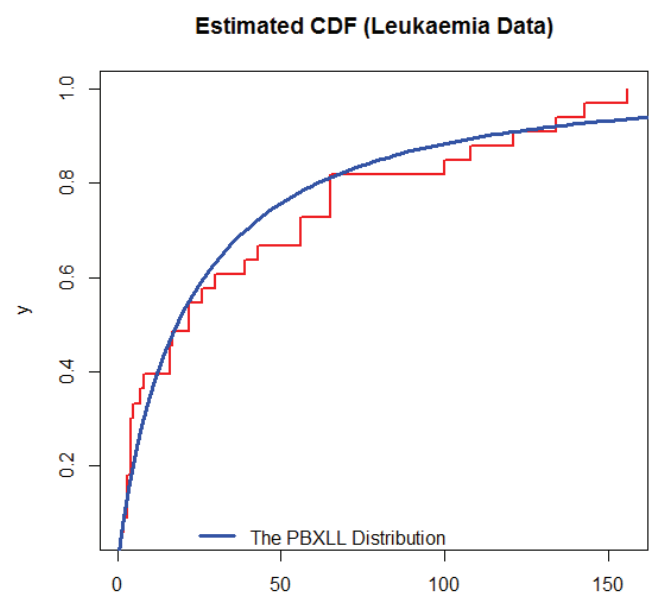

Data set IV.

Figure 4. Estimated CDFs 


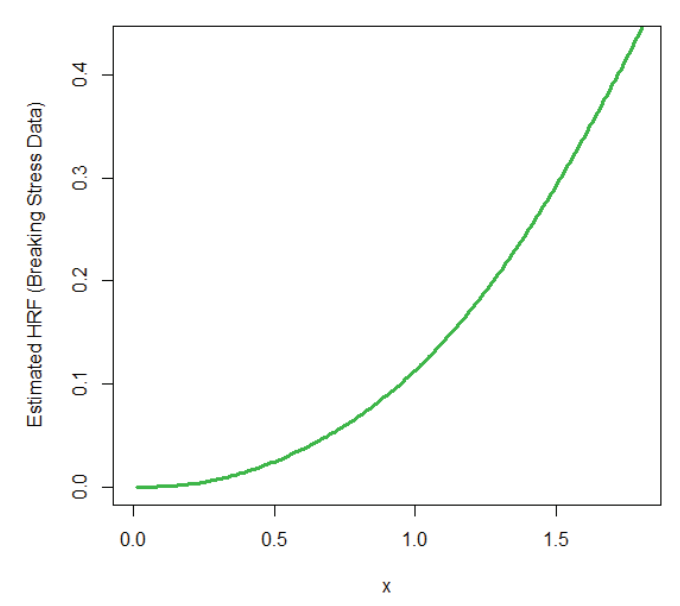

Data set $\mathbf{I}$.

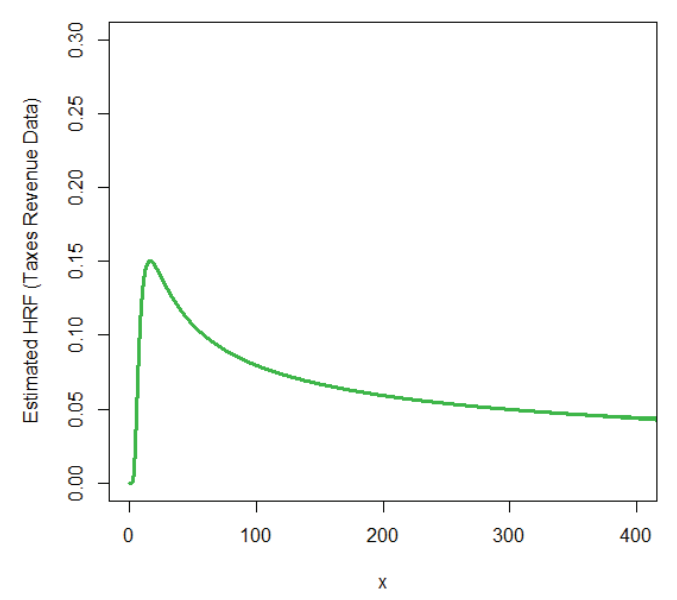

Data set III.

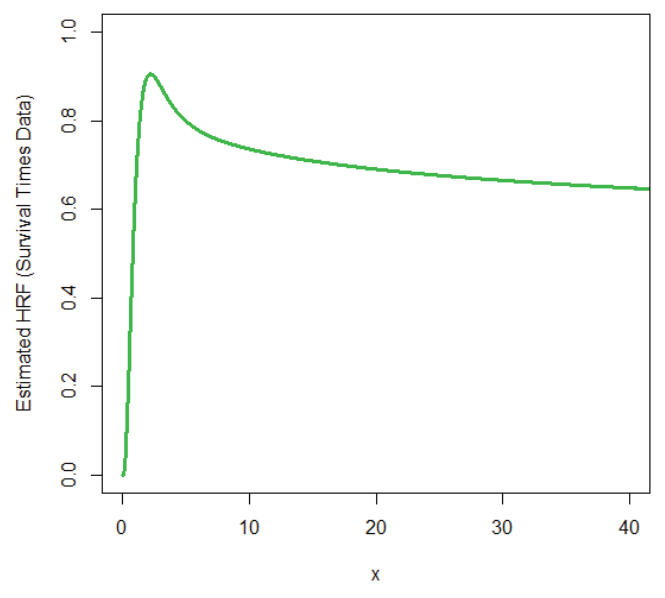

Data set II.

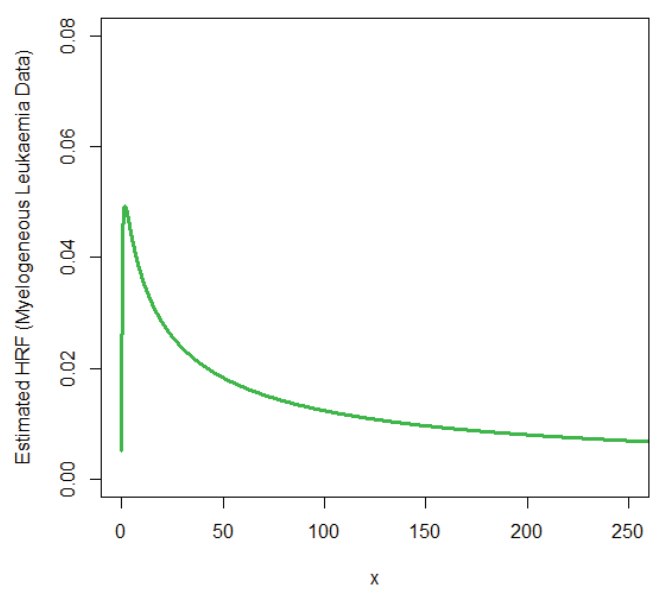

Data set IV.

Figure 5. Estimated HRFs 
P-P Plot for Breaking Stress of Carbon Fibres

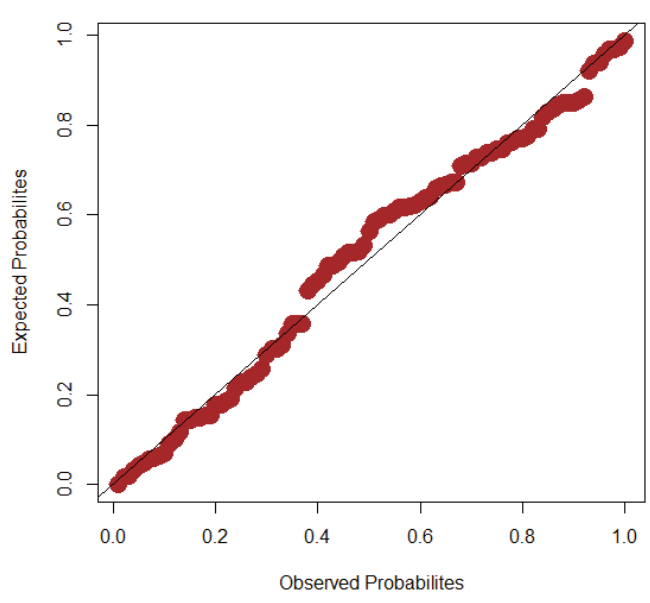

Data set I

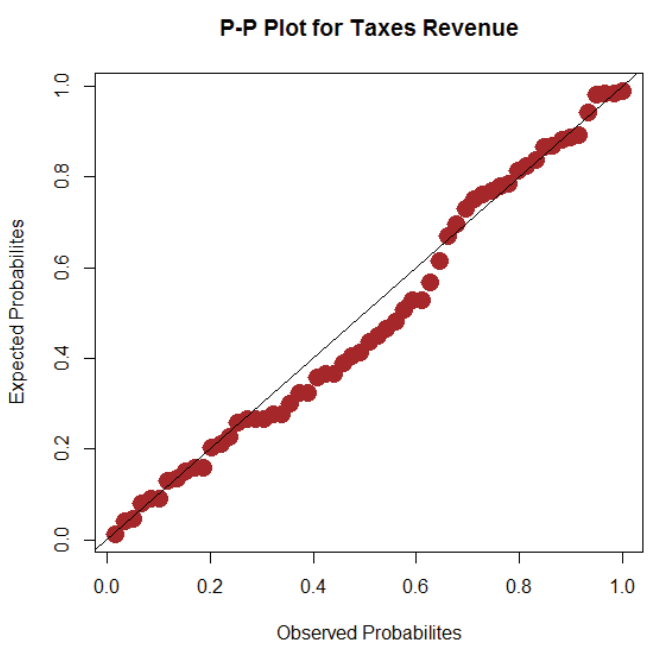

Data set III

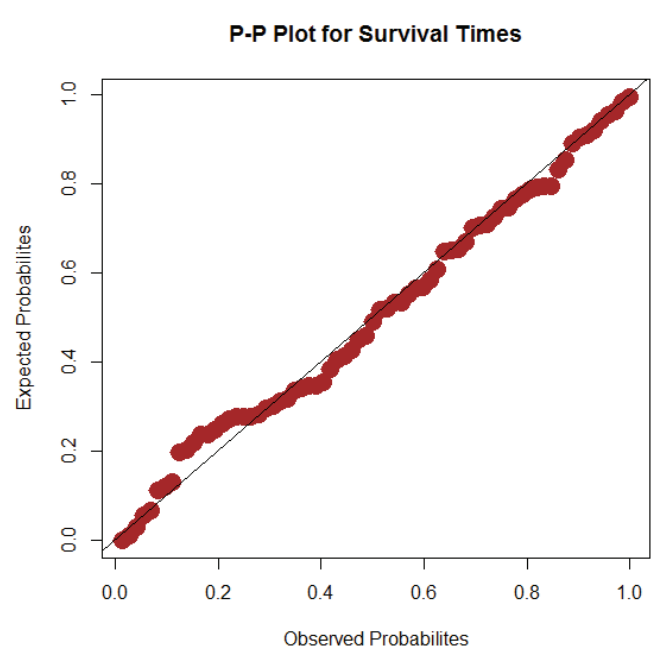

Data set II

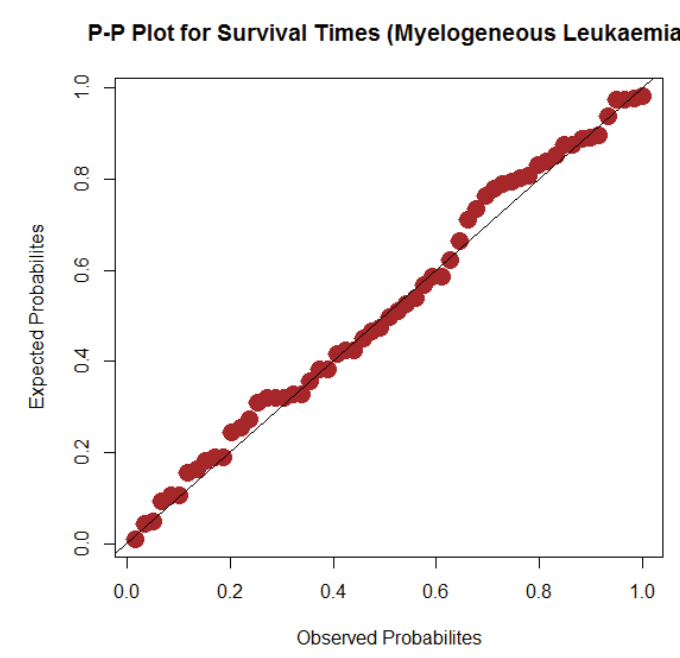

Data set IV

Figure 6. P-P plots 


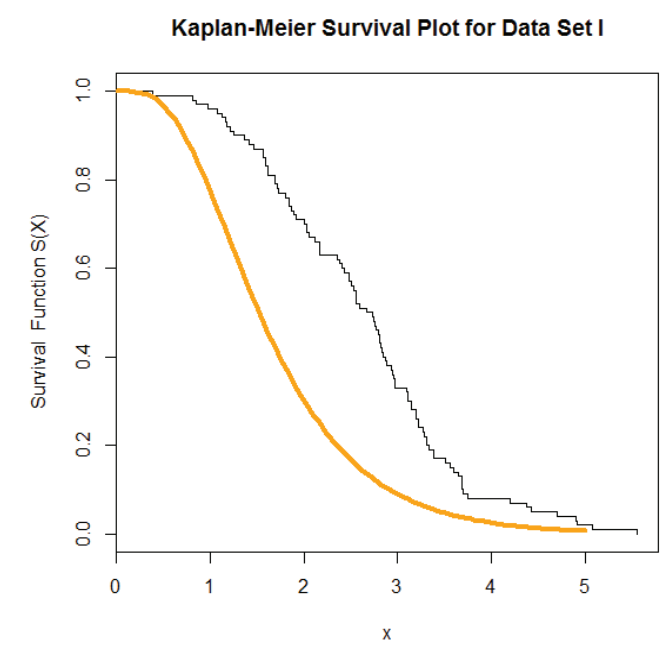

Data set $\mathbf{I}$.

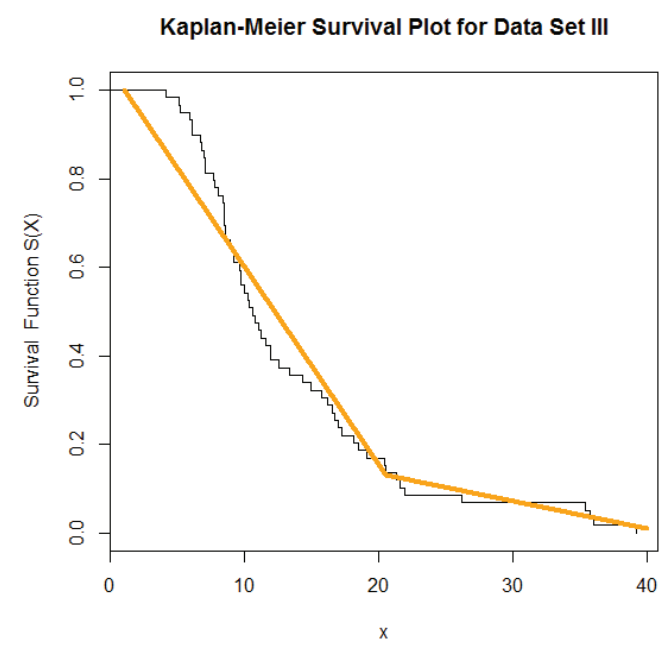

Data set III.

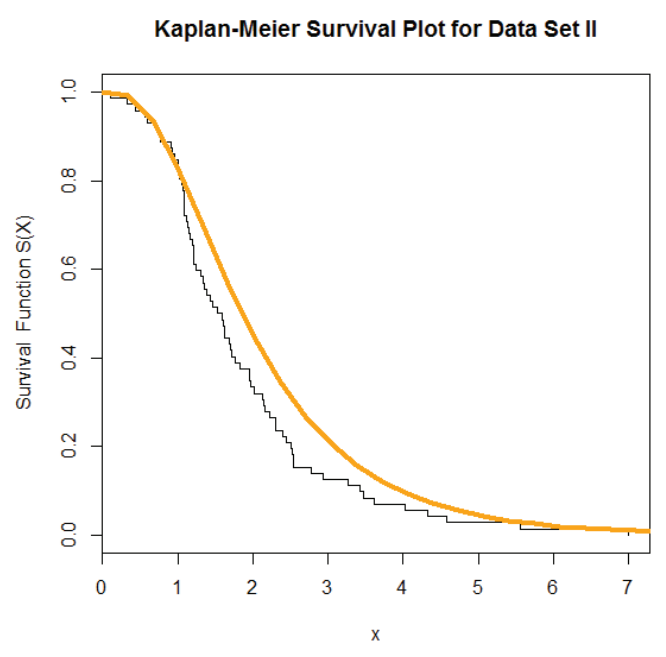

Data set II.

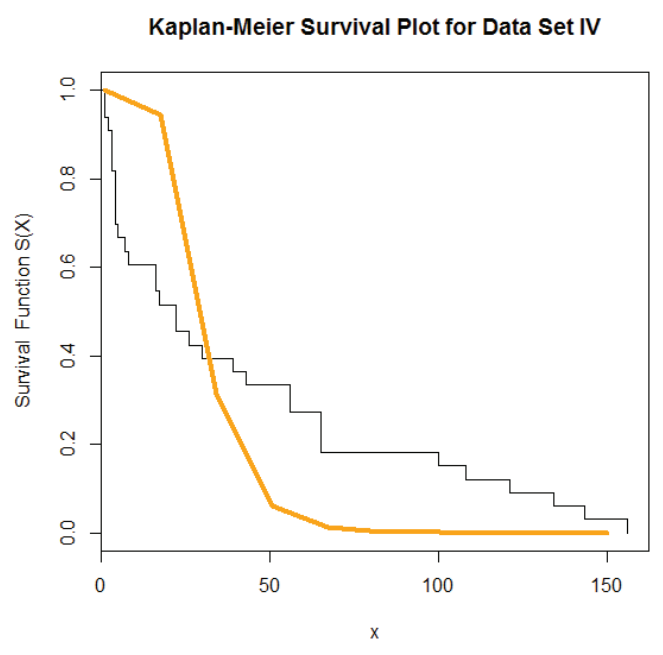

Data set IV.

Figure 7. Kaplan-Meier Survival plots

\section{Conclusions}

In this paper, we introduce a new Poisson-log-logistic distribution with a physical interpretation and capacious applications. Some essential properties are derived. Modeling of four real data sets are provided to illustrate the wide applicability of the new model in differnt fields like finance, reliability, economy and medicine. The new compound model is better than other well-known competitive models which have at least the same number of parameters.

\section{References}

Altun, E., Yousof, H. M., \& Hamedani G. G. (2018a). A new log-location regression model with influence diagnostics and residual analysis. International Journal of Applied Mathematics and Statistics, forthcoming.

Altun, E., Yousof, H. M., Chakraborty, S., \& Handique, L. (2018b). Zografos-Balakrishnan Burr XII distribution: regression modeling and applications, International Journal of Mathematics and Statistics, forthcoming.

Bjerkedal, T. (1960). Acquisition of resistance in Guinea pigs infected with different doses of virulent tubercle bacilli. American Journal of Hygiene, 72, 130-148.

Burr, I. W. (1942). Cumulative frequency functions. Annals of Mathematical Statistics, 13, 215-232. https://doi.org/10.1214/aoms/1177731607

Burr, I. W. (1973). Parameters fora general system of distributions to match a grid of $\alpha 3$ and $\alpha 4$. Communications in Statistics, 2, 1-21. https://doi.org/10.1080/03610927308827052 
Burr, I. W., \& Cislak, P. J. (1968). On a general system of distributions: I. Its curve-shaped characteristics; II. The sample median. Journalof the American Statistical Association, 63, 627-635.

Nichols, M. D., \& Padgett, W. J. (2006). A bootstrap control chart for Weibull percentiles. Quality and Reliability Engineering International, 22, 141-151. https://doi.org/10.1002/qre.691

Rodriguez, R. N. (1977). A guide to the Burr type XII distributions. Biometrika, 64, 129-134. https://doi.org/10.1080/03610918.2017.1377241

Tadikamalla, P. R. (1980). A look at the Burr and related distributions, International Statistical Review, 48, 337-344. https://doi.org/10.2307/1402945

Yousof, H. M., Altun, E., Ramires, T. G., Alizadeh, M., \& Rasekhi, M. (2018a). A new family of distributions with properties, regression models and applications, Journal of Statistics and Management Systems, 21, 163-188. https://doi.org/10.1080/09720510.2017.1411028

Yousof, H. M., Rasekhi, M., Altun, E., Alizadeh, M., Hamedani, G. G., \& Ali, M. M. (2018b). A new lifetime model with regression models, characterizations and applications. Communications in Statistics - Simulation and Computation, forthcoming. https://doi.org/10.1080/03610918.2017.1377241

\section{Appendix}

Data Set $\mathbf{I}\{0.98,5.56,5.080,0.39,1.570,3.19,4.90,2.930,2.85,2.77,2.760,1.73,2.48,3.680,1.08,3.220,3.75,3.22$, $3.70,2.74,2.730,2.50,3.60,3.110,3.27,2.87,1.47,3.11,4.91,1.590,1.18,2.480,2.03,1.690,2.43,3.390,3.56,2.830$, $3.68,2.0,3.510,0.85,1.61,3.280,2.95,2.810,3.150,1.920,1.840,1.22,2.170,1.61,4.420,2.40,4.20,2.350,1.410$, $1.59,1.12,1.69,2.79,1.89,1.87,3.39,3.33,2.55,3.68,3.19,1.71,1.25,4.70,2.88,2.96,2.55,2.59,2.97,3.15,2.67,3.31$, $2.81,2.56,2.17,2.12,3.09,2.97,1.57,2.17,4.38,2.03,2.82,2.53,3.310,2.38,1.360,0.81,1.170,1.84,1.80,2.050$, $3.650\}$.

Data Set II $\{0.10,0.33,0.440,0.56,0.590,0.72,0.740,0.77,0.920,0.93,0.960,1.0,1.0,1.020,1.05,1.070,02.02$, $1.360,1.39,1.440,1.46,1.530,1.59,1.60,1.63,1.630,1.680,1.71,1.720,1.760,1.83,1.95,2.130,2.15,2.160,2.220$, $2.3,2.310,2.4,2.450,2.51,2.530,7.0,1.080,1.08,1.080,1.09,1.120,1.13,1.150,1.16,1.20,1.21,1.220,1.220,1.24$, $1.30,1.34,1.960,1.970,2.540,2.54,2.780,2.930,3.270,3.420,3.47,3.610,4.020,4.32,4.580,5.55\}$.

Data Set III $\{5.90,20.40,14.9,16.20,17.2,12.50,10.30,11.2,6.10,8.4,11.0,11.6,7.80,6.10,9.2,10.20,9.6,8.50,8$, $9.2,26.20,21.9,16.70,21.30,13.30,8.5,21.6,18.5,5.1,6.7,17,8.60,9.7,39.2,35.7,15.70,9.7,10.0,4.1,36,35.40$, $14.3,8.50,10.6,19.10,20.5,7.1,7.70,18.10,16.50,11.9,7.0,8.6,11.90,5.2,6.80,8.90,7.1,10.80\}$.

Data set IV $\{65,121,4,39,143,56,26,22,1,1,156,100,65,17,7,16,22,3,4,2,3,8,4,3,30,134,16,108,5,65,56$, $4,43\}$.

\section{Copyrights}

Copyright for this article is retained by the author(s), with first publication rights granted to the journal.

This is an open-access article distributed under the terms and conditions of the Creative Commons Attribution license (http://creativecommons.org/licenses/by/4.0/). 